Erminio PIVA*

\title{
Due nuovi Orotrechus Müller del Vicentino (Veneto, Italia) e note sinonimiche (Coleoptera Carabidae Trechinae)
}

\begin{abstract}
Riassunto: Vengono qui descritti Orotrechus biancoi n. sp., di cavità del Bosco del Tretto (Schio, Vicenza) (Gruppo del Carega - Piccole Dolomiti - Pasubio) e Orotrechus euganeus perettoi n. ssp., di ambiente sotterraneo superficiale e grotte dei Monti Berici (Arcugnano e Longare, Vicenza). Lo studio di abbondante materiale ha potuto dimostrare l'ampia variabilità delle sottospecie descritte di Orotrechus stephani, permettendo così di stabilire le due seguenti nuove sinonimie: Orotrechus stephani roboretanus Müller, 1933=Orotrechus stephani stephani (Müller, 1913) e Orotrechus stephani perinii Tamanini, 1953=Orotrechus stephani stephani (Müller, 1913). Viene inoltre proposta una tabella dicotomica, opportunamente modificata, per il riconoscimento degli Orotrechus del gruppo stephani. Alcune note ecologiche, infine, vengono riportate per il nuovo taxon dei Monti Berici.
\end{abstract}

\begin{abstract}
Two new Orotrechus Müller from the Vicenza area (Veneto, Italy) and synonymic notes (Coleoptera Carabidae Trechinae). Orotrechus biancoi n. sp., from caves of the Bosco del Tretto (Schio, Vicenza) (Gruppo del Carega-Piccole Dolomiti-Pasubio) and Orotrechus euganeus perettoi n. ssp., from subterranean environment and caves of the Berici Mounts (Arcugnano and Longare, Vicenza), are here described. Studing the abundant material it has been demonstrated the great variability of the described subspecies of Orotrechus stephani, so allowing to establish the following two new synonymies: Orotrechus stephani roboretanus Müller, 1933=Orotrechus stephani stephani (Müller, 1913) and Orotrechus stephani perinii Tamanini, 1953=Orotrechus stephani stephani (Müller, 1913). A key for identification, properly modified, to the Orotrechus of the stephani species group, is also proposed. Finally, some ecological notes on the new taxon, inhabiting the Berici Mounts, are given.
\end{abstract}

Key words: Trechinae, Orotrechus, New species, New subspecies, Synonymies, Veneto, Italy.

\section{INTRODUZIONE}

Nel corso di un decennio di ricerche faunistiche in cavità del Vicentino (Veneto, Italia) il collega Dante Bianco, coadiuvato da altri soci del Gruppo Grotte CAI di Valdagno (Vicenza), ha potuto radunare vari esemplari di Orotrechus Müller che, comunicatimi in studio, si sono rivelati appartenere ad una nuova specie, più avanti descritta.

Numerosi altri individui di Orotrechus, raccolti in grotta e in ambiente endogeo dai componenti la Commissione Biospeleologica del Club Speleologico Proteo di Vicenza, giacevano da tempo in attesa di un accurato esame. La descrizione della nuova specie, cui ho accennato più sopra, mi ha offerto l'opportunità di studiare questi ulteriori reperti e di assegnarli ad un taxon inedito, ma in tal caso di rango sottospecifico.

Il materiale è conservato nelle collezioni del Museo Naturalistico Archeologico di Vicenza (MNAV), del Museo delle Scienze di Trento (MUSE), di Dante Bianco (Santorso, Vicenza) (CBI), di Achille Casale (Sassari) (CCA), di Pier Mauro Giachino (CGI) e di Erminio Piva (Vicenza) (CPI).
Orotrechus biancoi $\mathrm{n}$. sp.

Diagnosi. Un Orotrechus di medie dimensioni (Figg. 1-8), appartenente al "gruppo stephani", sensu Tamanini, 1953b e Vigna Taglianti, 1981, 1982, simile e affine, per habitus e dimensioni, a $O$. pominii Tamanini, 1953, ma distinto, da questo e dagli altri taxa congeneri, per l'apice del lobo mediano dell'edeago nettamente piegato ventralmente e per la conformazione della lamella copulatrice.

LOCALITÀ TIPICA. Veneto, Gruppo del Carega-Piccole Dolomiti-Pasubio. Schio (VI); Pozzo di San Patrizio (6542 V/VI), $460 \mathrm{~m}$.

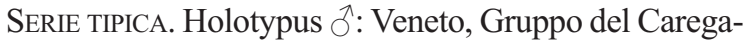
Piccole Dolomiti-Pasubio. Schio (VI); Pozzo di San Patrizio (6542 V/VI), 460 m., 1.XII.2007, D. Bianco \& N. Righetto leg. (CPI). Paratipi, 48 exx. (16 えぇ 32 우): Veneto, Gruppo del Carega-Piccole Dolomiti-Pasubio, Schio (VI), Pozzo di San Patrizio (6542 V/VI), 460 m, 11.I.2001, D. Bianco leg., 1 क (CBI); ibidem, 30.XII.2001, F. Pretto \& N. Righetto leg., 1 구 4 우우

*Erminio Piva, Via De Amicis 59, 36100 Vicenza, Italia. E-mail: erminiopiva1@virgilio.it 
(CBI, CPI); ibidem, 2.I.2003, D. Bianco, F. Casa \& N. Righetto leg., 1 के (CBI); ibidem, 20.IV.2003, N. Righetto leg., 1 q (CBI); ibidem, 25.VIII.2003, F. Casa \& N. Righetto leg., 4 우 (CBI, CPI); ibidem, 2.VI.2004, D. Bianco \& N. Righetto leg., 1 \& (CPI); ibidem, 27.V.2007, D. Bianco, E. Piva \& N. Righetto leg., 1 đ (CPI); ibidem, 1.XII.2007, D. Bianco \& N. Righetto leg., 2 추 3 우우 (CBI, CPI); ibidem,

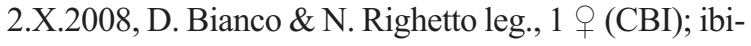
dem, 21.IX.2010, D. Bianco \& N. Righetto leg., 1 (MNAV); Veneto, Gruppo del Carega-Piccole Dolomiti-Pasubio, Schio (VI), loc. S. Maria del Tretto, Grotta Busa Freda (non catastata), 395 m, 5.XI.2003, F. Casa \& N. Righetto leg., 1 o 1 (CBI); ibidem, 30.IV.2004, F. Casa \& N. Righetto leg., 1 ( (CBI); ibidem, 18.XI.2006, D. Bianco \& F. Casa leg., 1 + (CPI); ibidem, 11.XII.2006, D. Bianco leg., 1 \& (CPI); Veneto, Gruppo del Carega-Piccole Dolomiti-Pasubio, Schio (VI), loc. S. Ulderico, Voragine 1 del Bosco delle Mole (643 V/VI), 700 m, 25.X.2003, D. Bianco \& N. Righetto leg., 1 \& (CBI); Veneto, Gruppo del Carega-Piccole Dolomiti-Pasubio, Schio (VI), loc. S. Ulderico, Abisso Righetto (non catastato), 640 m, 5.IV.2009, L. Dalle Tezze, A. De Munari \& A. Radin leg., 10 (CPI); ibidem, 2.X.2009, D. Bianco \& A. De Munari leg., 2 초 2 우우 (CBI, CPI); ibidem, 26.IX.2010, D. Bianco $\&$ A. De Munari leg., 1 수 2 우 (CBI, CGI, CPI); ibi-

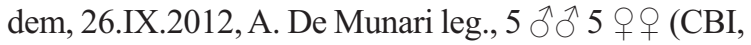
CCA, CPI); ibidem, 30.III.2014, L. Dalle Tezze, D. Sbicego, M. Tomasi, P. Urbani \& G. Zarantonello leg., 1 ऽ 우 (CBI, CPI, MUSE).

DeRIVATIO NOMINIS. Dedico con piacere la nuova specie all'amico e collaboratore Dante Bianco (Santorso, Vicenza), attivo ricercatore di microfauna ipogea e autore di gran parte dei campionamenti del nuovo taxon.

Descrizione Dell'Holotypus $\widehat{\jmath}$. Corpo a facies afenopsiana (Fig. 1), con appendici allungate ma non gracili, lungo $4.26 \mathrm{~mm}$ (misurato dall'apice delle mandibole all'apice elitrale), di colore testaceo chiaro, ricoperto da fitta pubescenza semieretta, più allungata sul capo; tegumenti con finissima microscultura a maglie poligonali, più trasverse sul cranio.

Capo largo $0.67 \mathrm{~mm}$, lungo $0.80 \mathrm{~mm}$ (dal punto più avanzato del margine anteriore del labbro superiore al restringimento collare); il rapporto lungh./largh. max. è 1.19. Occhi assenti, tempie moderatamente convesse, pubescenti; solchi frontali in- completi, bene incisi, posteriormente svaniti ben prima della seconda setola sopraorbitale. Sono presenti due setole sopraorbitali per lato, inserite su due linee subparallele. Antenne lunghe $2.84 \mathrm{~mm}$ (rapporto lungh. antenne/lungh. corpo $=0.67$ ), con il primo articolo ispessito, più lungo del secondo ma meno del terzo; dal terzo al quinto gradatamente più allungati, sesto lungo quanto il quarto, decrescenti dal sesto all'ottavo, nono più lungo dell'ottavo ma poco più lungo del decimo, undicesimo più lungo di tutti. Apparato boccale senza particolari caratteristiche.

Protorace tanto lungo quanto largo $(0.69 \mathrm{~mm})$, a lati arrotondati nel terzo anteriore, poi progressivamente ristretti fino alla base, non sinuati, con angoli posteriori retti; doccia marginale stretta e regolare,

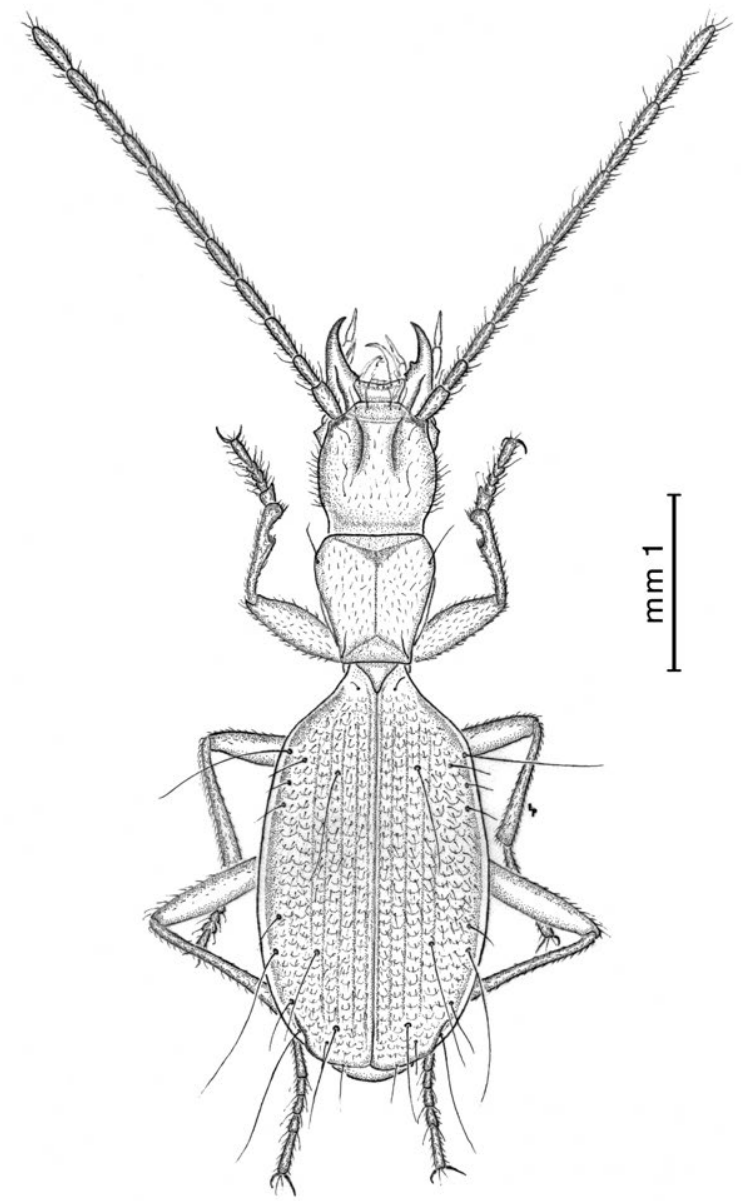

Fig. 1. Orotrechus biancoi n. sp., holotypus $\delta^{\Uparrow}$ : habitus. 
propleure e proepisterni per breve tratto visibili dall'alto. Setole pronotali presenti, le anteriori molto sviluppate, lunghe circa tre volte le posteriori.

Elitre ellittiche allungate, con omeri arrotondati, senza angolosità evidente, carena apicale molto sviluppata, delimitante un lobo piuttosto ampio, doccia marginale relativamente larga; poco incise ma distinguibili le quattro strie interne, le altre parzialmente o compiutamente svanite. Chetotassi: setola basale disposta di lato e quasi a livello del vertice dello scutello; serie discale di due setole, inserite sulla quarta interstria (la prima) e sulla quinta interstria (la seconda) e disposte in modo diverso sulle due elitre: l'anteriore a circa un quarto dalla base elitrale, la posteriore a quasi tre quarti, sull'elitra destra, entrambe un po' più avanzate su quella sinistra. Serie ombelicata con gruppo omerale disaggregato: la prima setola, interna, più vicina alla seconda che non alla terza, quest'ultima più vicina alla quarta che non alla prima. Setole quinta e sesta molto scostate dal gruppo omerale; la distanza tra loro è circa quella che intercorre tra la seconda e la terza, sull'elitra sinistra, ma uguale alla distanza tra la terza e la quarta sull'elitra destra. Settima verso l'ultimo sesto elitrale, ottava a lato della carena apicale, a circa metà del suo sviluppo. Triangolo apicale: setola anteriore, maggiormente sviluppata, sulla terza interstria; l'esterna, di poco scostata lateralmente e di minore lunghezza, la marginale all'apice elitrale, più ravvicinata alla stria suturale della setola anteriore, un po' più corta dell'esterna.

Zampe pubescenti; primo protarsomero dilatato e dentato internamente, quarto articolo dei protarsi e dei mesotarsi munito inferiormente di un tubercolo apicale dotato di lunghi faneri.

Apparato copulatore maschile: edeago robusto, lungo $0.71 \mathrm{~mm}$ dalla base all'apice. In visione laterale (Fig. 2) risulta poco incurvato, con bulbo basale non distinto; il margine ventrale è pressoché rettilineo e l'apice è rivolto verso il lato ventrale. Carena sagittale assente (ma presente in un paratipo: vedi oltre). In visione dorsale (Fig. 3) il lobo mediano presenta i lati regolarmente incurvati fin quasi alla sommità che è moderatamente ristretta. Parameri ad apice arrotondato, il destro più ampio, muniti di cinque setole, quattro maggiori ed una più breve; quest'ultima diversamente posizionata, preapicale nel paramero sinistro, dorso-apicale nel destro. Sono presenti alcuni pori non setigeri. Endofallo con lamella copulatrice molto grande (Figg. 4 e 5), caudata e svasata alla base, raccordata con l'apice da un setto obliquo compresso lateralmente.

DESCRIZIONE DEI PARATIPI E VARIABILITÀ. Lunghezza del corpo (dall'apice delle mandibole all'apice elitrale):

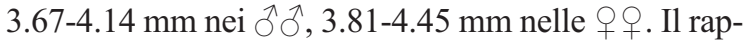
porto lunghezza antenne/lunghezza corpo è di 0.64-0.66

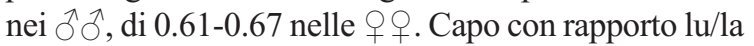

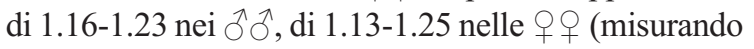
la lunghezza del capo dal punto più avanzato del margine anteriore del labbro superiore al restringimento collare). Il protorace, pressoché tanto lungo quanto

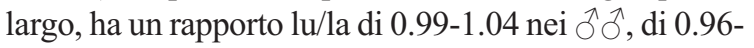
1.04 nelle + 0 ; in soli quattro casi i lati sono debol-

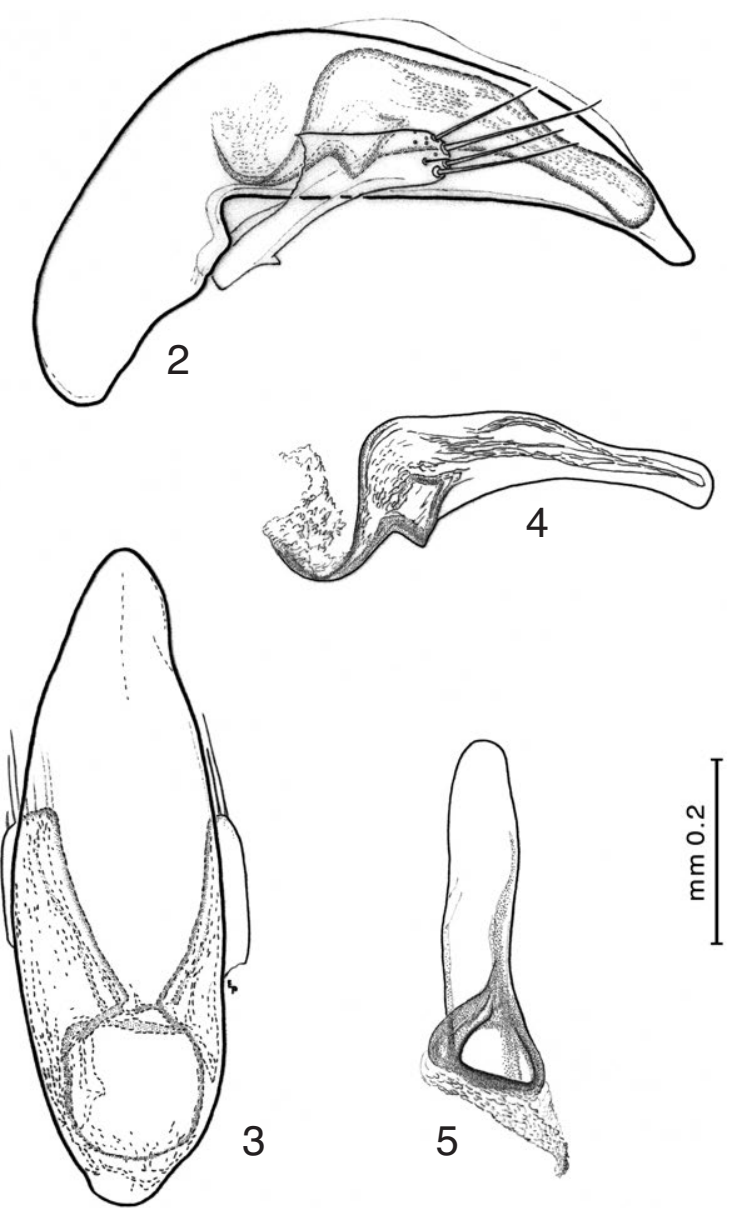

Figg. 2-5. Orotrechus biancoi n. sp., holotypus đ̊ : 2. edeago, in visione laterale; 3 . idem, in visione dorsale; 4. lamella copulatrice, in visione laterale; 5 . idem, in visione ventrale. 
mente e brevemente sinuati prima degli angoli posteriori e questi ultimi talvolta sono smussati, anziché vivi, mentre in alcuni esemplari sono ottusi, non retti, a causa di una smarginatura della base del pronoto. Elitre e relativa chetotassi senza particolarità di rilievo. Nei maschi l'apparato copulatore è generalmente conforme a quello dell'holotypus; soggetti a variabilità solo il numero (5 o 6) e la lunghezza delle setole dei parameri. In un paratipo l'edeago è munito di carena sagittale. Armatura genitale femminile con gonostili $1.2 \div 1.6$ volte più lunghi che larghi, ad apice arrotondato, provvisti di due setole tergali, la distale sempre molto robusta; fossetta preapicale ampia, munita di due setole e di due (raramente tre o quattro) bastoncelli sensoriali. Presenti numerosi pori $(9 \div 19)$, alcuni recanti una microsetola. Nelle Figg. 6-8 è rappresentata l'armatura genitale femminile di un paratipo topotipico.

DisTRIBUZIONE, ECOLOGIA. Le quattro cavità ospitanti il nuovo taxon sono tutte ubicate in un' area nota come Bosco del Tretto, a nord della città di Schio (Vicenza); le due a bassa quota, la Grotta Busa Freda (395 m) e il Pozzo di San Patrizio, località tipica $(460 \mathrm{~m})$ sono ad andamento orizzontale, mentre le due a maggiore altitudine, l'Abisso Righetto (640 m) e la Voragine 1 del Bosco delle Mole (700 m), si sviluppano verticalmente. Sono grotte oligotrofiche caratterizzate dalla presenza di consistenti depositi fangosi. Le catture del nuovo Orotrechus, ottenute mediante l'uso di trappole a caduta, sono avvenute principalmente sotto depositi franosi, costituiti da sassi infossati in strati fangosi, formanti tra loro un vasto sistema di fessure.

\section{OSSERVAZIONI SUGLI OROTRECHUS}

\section{DEL "GRUPPO STEPHANI" E NOTE SINONIMICHE}

Le specie di Orotrechus finora note del "gruppo stephani" erano due, una politipica, O. stephani, descritta da Müller nel 1913, alla quale sono state aggiunte in seguito tre sottospecie, $O$. s. roboretanus Müller, 1933, O. s. perinii Tamanini, 1953 e $O$. s. prenottoi Daffner, 1986 (quest'ultima in origine indicata come specie distinta); la seconda era $O$. pominii Tamanini, 1953.

L'accurato esame morfometrico effettuato su tutti gli individui di $O$. stephani s.l. a mia disposizione (provenienti sia da raccolte personali sia da Musei e privati) mi ha dato l'opportunità di meglio definire i limiti di variabilità dei vari caratteri diacritici utilizzati nelle descrizioni.
Ho esaminato complessivamente 118 exx. (56 ふ઼ 62 우우) di Orotrechus stephani stephani, tutti provenienti da cavità venete e trentine dell'Altopiano dei Sette Comuni (o Altopiano di Asiago). Oltre a reperti della località tipica, la Grotta di Costalta (14 VT/TN), $1691 \mathrm{~m}$, Borgo Valsugana (TN), segnalo le seguenti stazioni inedite: Grotta Lago Bianco (non catastata), 1370 m ca., Asiago (VI); Grotta della Colonnetta (6376 V/VI), 1450 m, Asiago (VI); Senza Elle (7138 V/VI), 1445 m, Asiago (VI); Abisso del Grankio (non catastata), $1350 \mathrm{~m}$ ca., Asiago (VI); LSG 6 (6565 V/VI), $1580 \mathrm{~m}$, Asiago (VI); Abisso Canaglia (2762 V/VI), 2055 m, Asiago (VI); Abisso di Busa del Ghiaccio (2057 V/VI), 1548 m, Foza (VI); Cogol dei Siori (600 V/VI), $147 \mathrm{~m}$, Valstagna (VI); Spaluga di Lusiana (86 V/VI), $1100 \mathrm{~m}$, Lusiana (VI).

Di Orotrechus s. roboretanus ho studiato 38 esemplari, provenienti da sei grotte veneto-trentine (delle quali le ultime quattro elencate sono inedite)
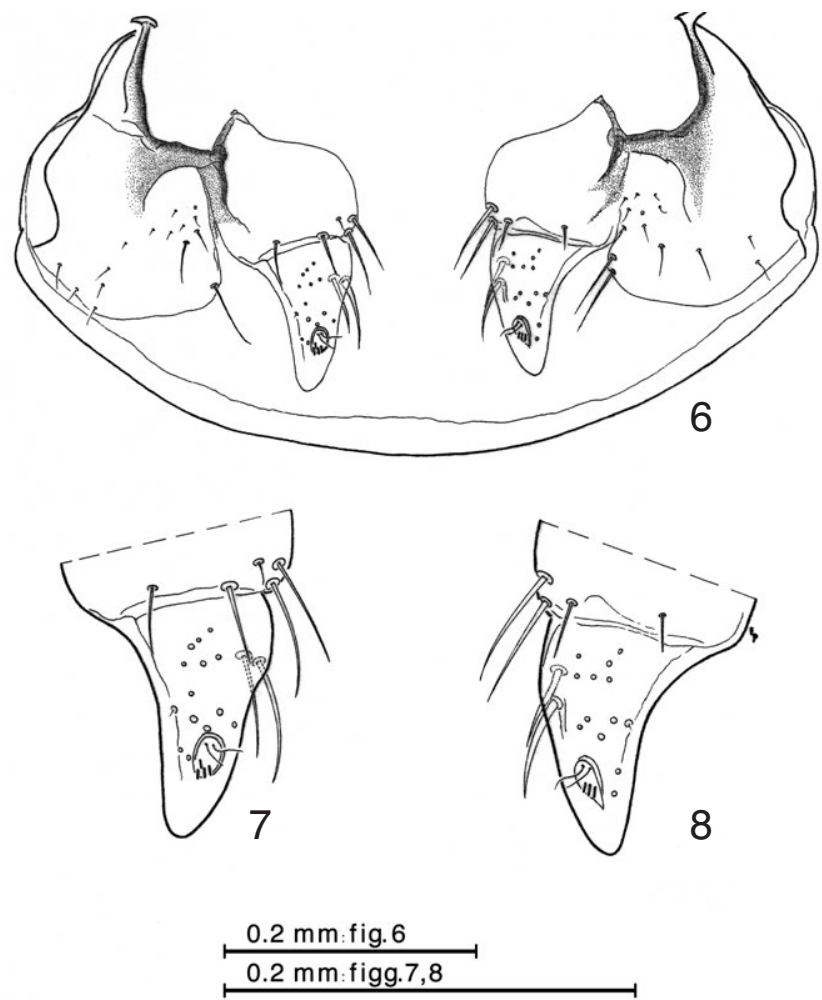

Figg. 6-8. Orotrechus biancoi n. sp., paratypus $q$ (Pozzo di San Patrizio, località tipica): 6. armatura genitale femminile in visione ventrale; 7-8. gonostili a maggiore ingrandimento. 

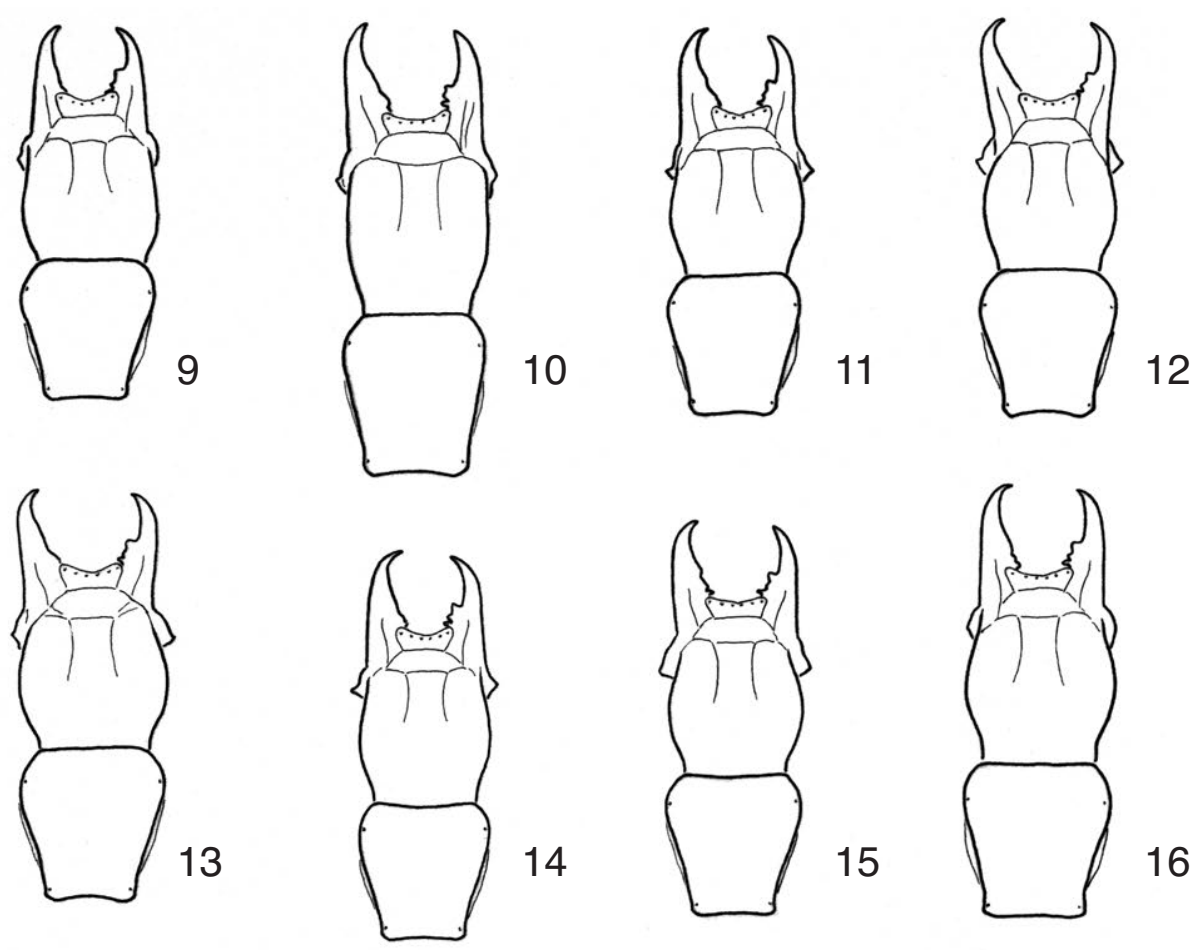

16
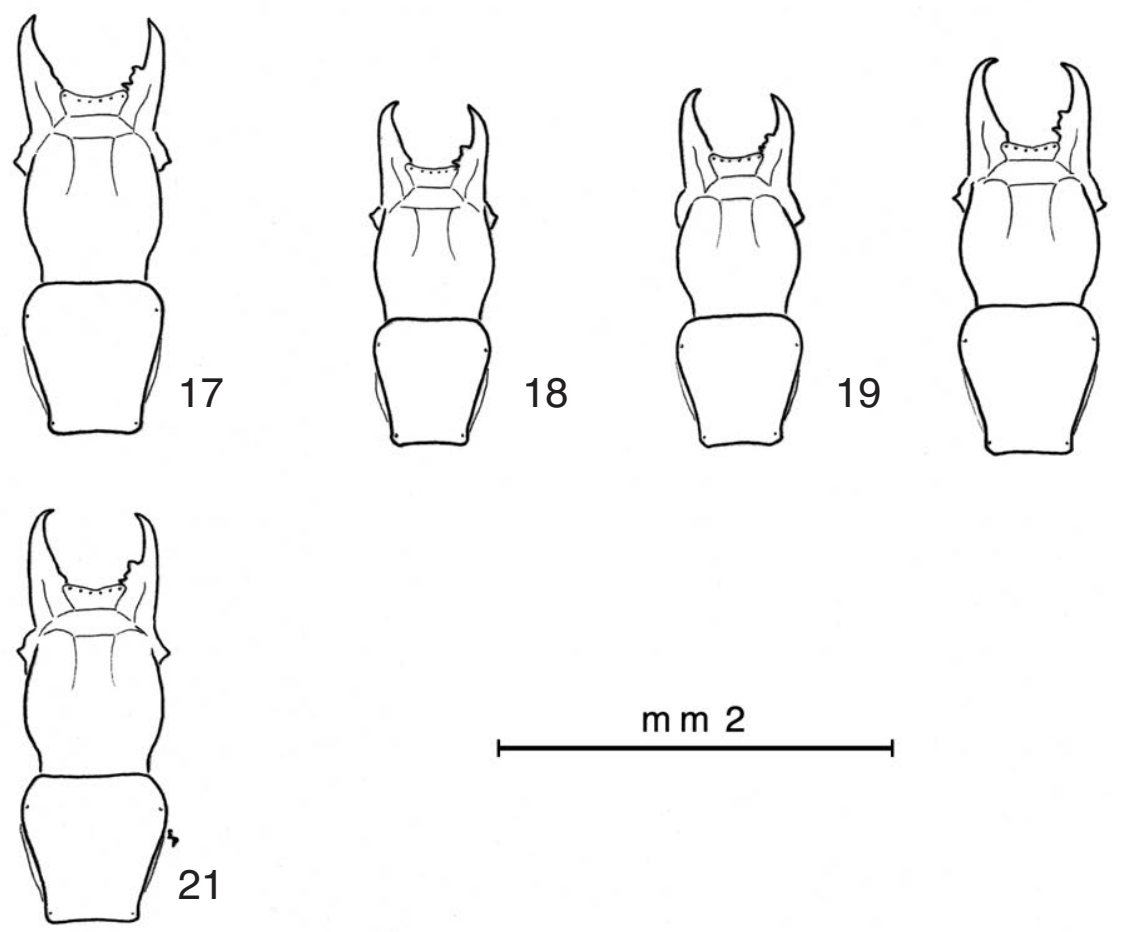

$\mathrm{m} m 2$

Figg. 9-21. Orotrechus stephani stephani (Müller), variabilità interpopolazionale; capo e pronoto di individui delle seguenti cavità: 9. ex. $q$ del Cogol dei Siori; 10. ex. $q$ della Grotta di Costalta; 11. ex. $\subsetneq$ della Grotta G.B. Trener; 12. ex. $q$ e 18-19. exx.

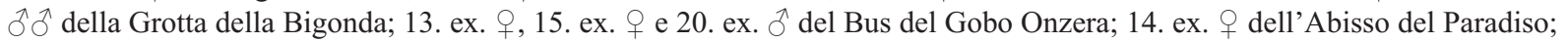

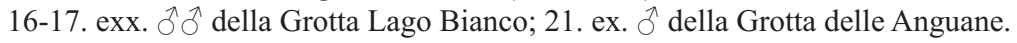



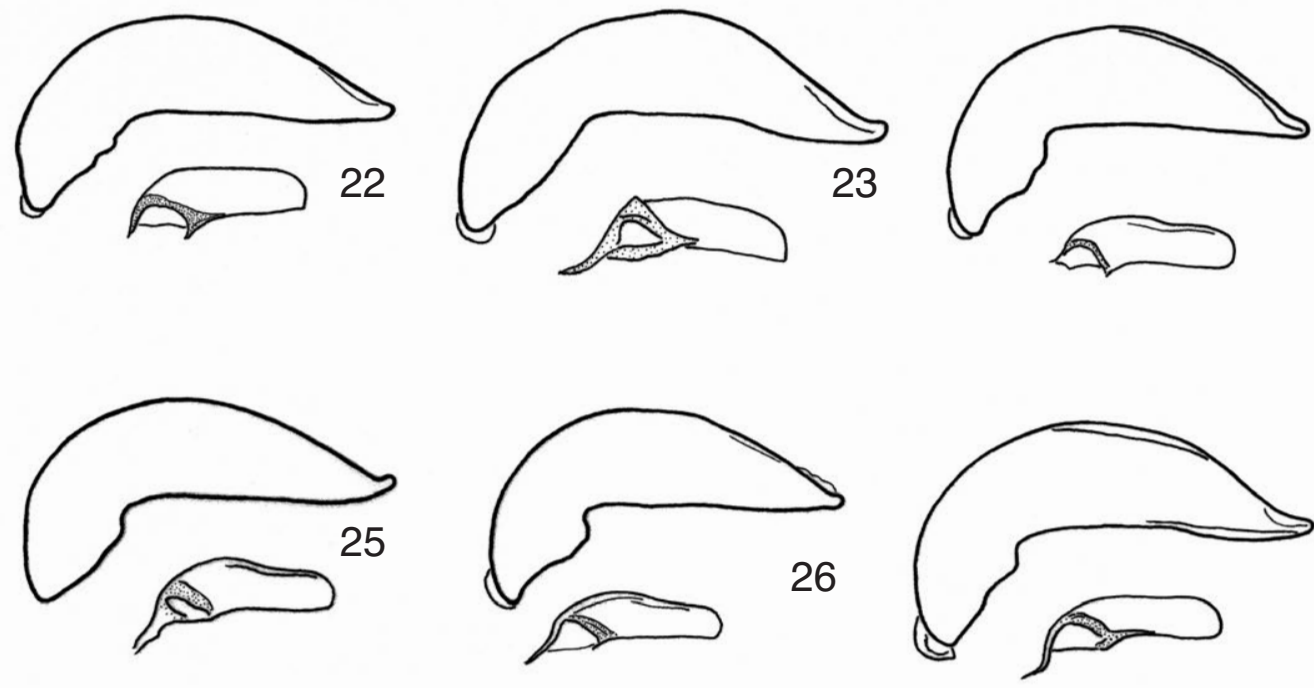

27
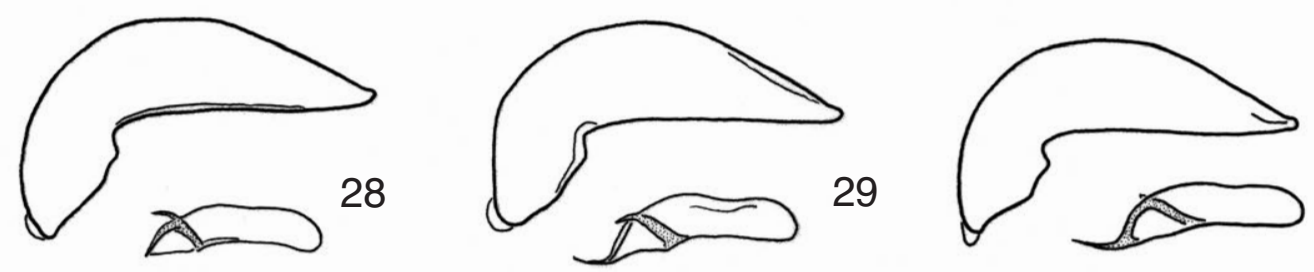

30
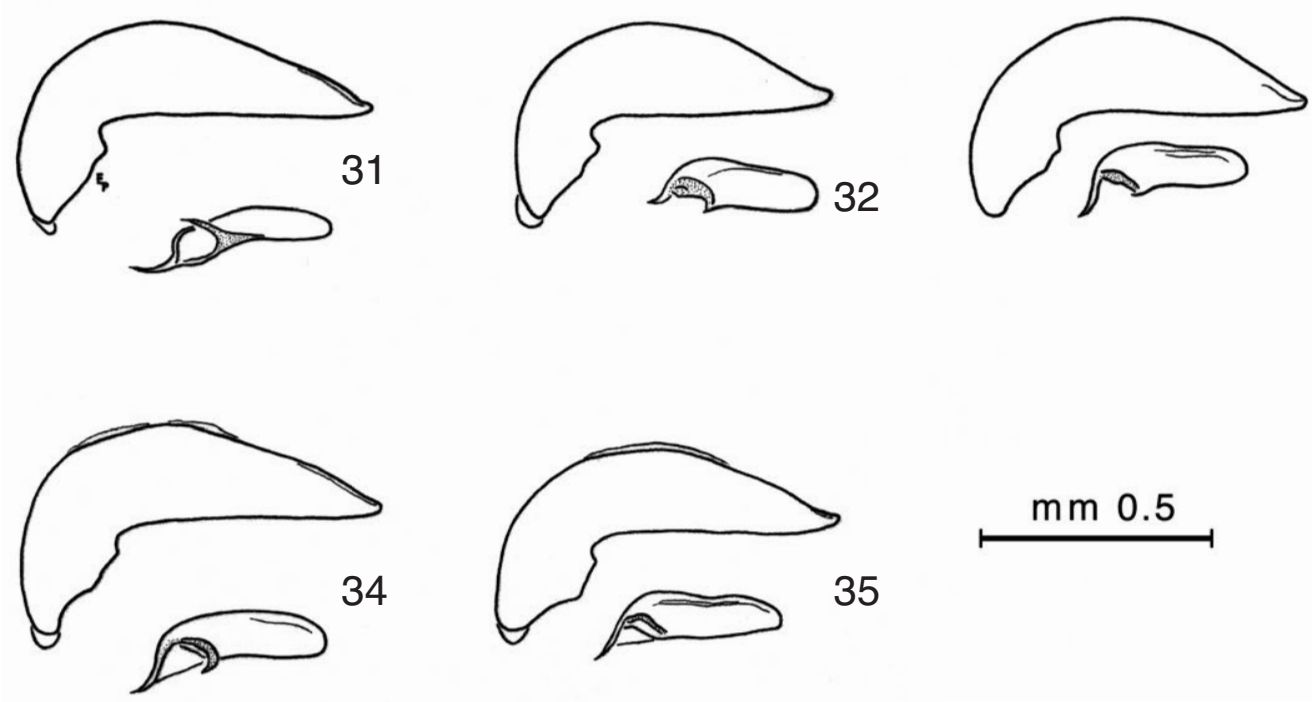

$\mathrm{mm} 0.5$

Figg. 22-35. Orotrechus stephani stephani (Müller), variabilità interpopolazionale; lobo mediano dell'edeago e lamella copulatrice, in visione laterale, di individui delle seguenti cavità: 22-23. Grotta di Costalta; 24-25. Grotta Lago Bianco; 26-27. Grotta della Colonnetta; 28. Senza Elle; 29. Abisso del Grankio; 30. Spaluga di Lusiana; 31-32. Grotta della Bigonda; 33. Grotta G.B. Trener; 34-35. Bus del Gobo Onzera. 
ubicate negli Altipiani di Tonezza-Folgaria e Vigolana, i.e. Bus del Gobo Onzera (207 VT/TN), 1295 m, Folgaria (TN); Abisso Malga Melegna (626 VT/TN), $1636 \mathrm{~m}$, Folgaria (TN); Abisso del Paradiso (870 V/VI), $1500 \mathrm{~m}$, Tonezza del Cimone (VI); Grotta delle Anguane (523 V/VI), $796 \mathrm{~m}$, Tonezza del Cimone (VI); Buso del Sasso (1219 V/VI), 860 m, Arsiero (VI); Buso Alto del Sasso (4047 V/VI), 950 m, Arsiero (VI). In letteratura (Müller, 1933) è citato anche il Bus del Bilbom, dove furono raccolti i resti del primo esemplare di questa entità.

Inoltre ho esaminato l'olotipo e due paratipi di $O$. s. perinii, concessimi in studio dal Museo delle Scienze di Trento e altri 21 esemplari, provenienti dalla Grotta della Bigonda (243 VT/TN), 450 m, Grigno (TN) e dalla Grotta G.B. Trener (o Grotta del Calgeron) (244 VT/TN), $450 \mathrm{~m}$, Grigno (TN).

L'esito dello studio di questi tre taxa ha messo in evidenza la notevole variabilità della specie, a tal punto da suggerire un significativo cambiamento nel
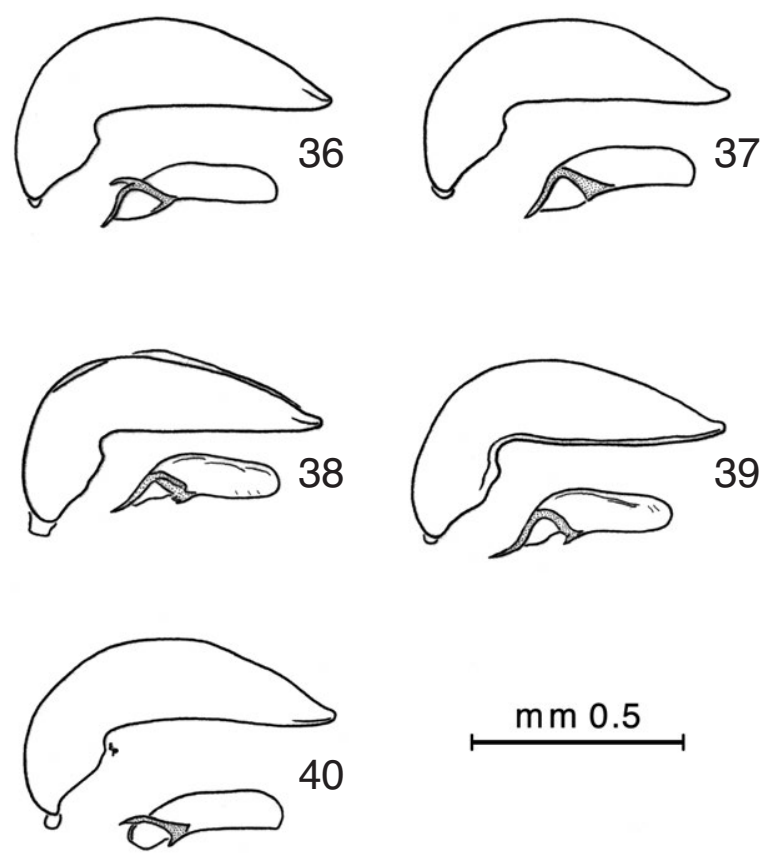

$\mathrm{mm} 0.5$

Figg. 36-40. Orotrechus stephani stephani (Müller), variabilità interpopolazionale; lobo mediano dell'edeago e lamella copulatrice, in visione laterale, di individui delle seguenti cavità: 36. Abisso del Paradiso; 37. Buso Alto del Sasso; 38-39. Grotta delle Anguane; 40. Abisso Malga Melegna. suo assetto tassonomico, come dirò più avanti, dopo aver analizzato i vari caratteri differenziali impiegati nelle descrizioni.

Per questa trattazione ho scelto il dettagliato, anche se datato, lavoro di Tamanini (1953b); tutti i caratteri messi a confronto dall'autore nelle sue tavole dicotomiche sono stati, qui di seguito, a mia volta riesaminati.

Morfologia esterna. La lunghezza del corpo (misurata dall'apice delle mandibole all'estremità delle elitre) è $4.24 \div 5.05 \mathrm{~mm}$ in $O$. s. stephani, $4.21 \div 5.14 \mathrm{~mm}$ in O. s. roboretanus, $4.03 \div 4.47 \mathrm{~mm}$ in $O$. s. perinii. I rispettivi rapporti lunghezza/larghezza capo (lunghezza misurata, per uniformità con il già citato contributo di Tamanini, dal margine anteriore del pronoto all'apice delle mandibole) sono $1.90 \div 2.04$ (media: 1.97), $1.86 \div 2.02$ (media: 1.95 ) e $1.88 \div 1.93$ (media: 1.91) nei $\hat{\sigma}^{\widehat{T}}{ }^{\top} ; 1.86 \div 2.10$ (media: 1.97 ), $1.80 \div 2.07$ (media: 1.95 ) e $1.86 \div 1.99$ (media: 1.92) nelle 우우. I rapporti lunghezza/larghezza pronoto sono rispettivamente $0.94 \div 1.10$ (media: 1.04), $0.99 \div 1.07$ (media: 1.02) e $1.01 \div 1.05$ (media: 1.03 ) nei ô 0 ; $0.97 \div 1.09$ (media: 1.02), $0.98 \div 1.04$ (media: 1.02 ) e $1.01 \div 1.09$ (media: 1.05 ) nelle $q$ ㅇ․

L'esame biometrico dimostra l'inaffidabilità, quali caratteri differenziali, dei rapporti fra lunghezza e larghezza sia del capo, sia del pronoto. Esemplari con capo a tempie rigonfie si ritrovano, frammisti ad individui "normali", in popolazioni riferibili a tutti e tre i taxa (Figg. 9-21).

Analogamente poco significativi risultano $i$ rapporti tra la massima larghezza delle elitre e la larghezza omerale, rispettivamente $1.16 \div 1.32,1.16 \div 1.30$ e $1.18 \div 1.26$.

È ritenuta importante da Tamanini (1953b) anche la posizione della $2^{\mathrm{a}}$ setola discale rispetto alla $5^{\mathrm{a}} \mathrm{e}$ alla $6^{a}$ della serie ombelicata; nel materiale da me studiato tale setola è solitamente posizionata tra le due della serie ombelicata, ma in circa un terzo dei casi è situata oltre la $6^{\mathrm{a}}$, in modo casuale e in varie popolazioni.

Edeago. Lobo mediano (Figg. 22-40), in visione laterale, variabilissimo nelle tre sottospecie: talvolta con apice diritto, talaltra rivolto verso il lato dorsale. Ho riscontrato entrambi i casi a tutti i livelli, intra- e interpopolazionali.

Del tutto simili tra loro anche i rapporti tra lunghezza dell'edeago e lunghezza del corpo, rispettivamente $0.16 \div 0.18,0.15 \div 0.18$ e $0.16 \div 0.19$. 
Lamella copulatrice, in visione laterale, di forma incostante, più o meno stretta, inferiormente troncata oppure no; inutilizzabile per distinguere tra loro i tre taxa.

A conclusione di questa analisi morfologica, che come risultato finale ha evidenziato l'inconsistenza di tutti i caratteri impiegati per differenziare le tre sottospecie, non mi rimane che proporre le seguenti sinonimie: Orotrechus stephani roboretanus Müller, 1933=Orotrechus stephani stephani (Müller, 1913) n. syn. Orotrechus stephani perinii Tamanini, 1953=Orotrechus stephani stephani (Müller, 1913) n. syn.

Dopo queste due importanti variazioni nomenclatoriali, mi sembra opportuno proporre alcune considerazioni conclusive sul complesso delle popolazioni di questa polimorfa sottospecie.
Il suo areale di distribuzione (Fig. 41) comprende grotte e abissi veneto-trentini degli Altipiani di Tonezza-Folgaria e Vigolana (con limite occidentale situato presso l'abitato di Serrada, Folgaria, Trento) e dell'Altopiano dei Sette Comuni (con limite orientale nella Valsugana, a Oliero, Valstagna, Vicenza). Procedendo da est ad ovest ho notato una generale, ma non costante, tendenza degli esemplari ad avere le strie elitrali meno evidenti; in ogni stazione, tuttavia, queste sono più marcate negli individui di sesso femminile. Il protorace risulta a lati meno evidentemente sinuati (da non sinuati o debolmente sinuati) negli esemplari dell'Altopiano dei Sette Comuni, rispetto a quelli delle popolazioni occidentali, ma anche in questo caso in modo incostante.

Ho notato, inoltre, che alle quote inferiori (le risorgenze Grotta della Bigonda, Grotta G.B. Trener e

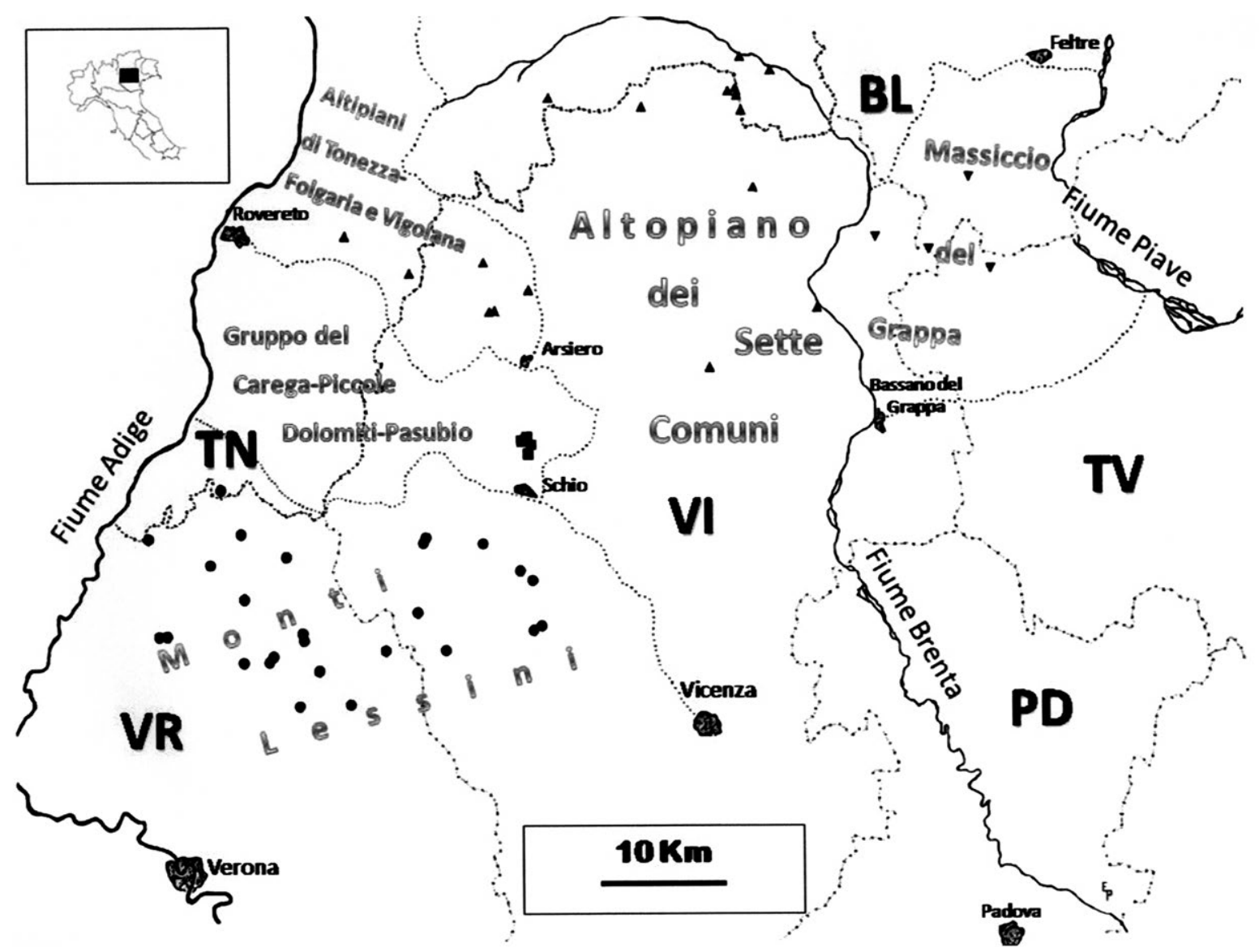

Fig. 41. Cartina di distribuzione degli Orotrechus del "gruppo stephani”: $\mathbf{\Delta}$ - Orotrechus stephani stephani (Müller); $\boldsymbol{\nabla}$ - Orotrechus stephani prenottoi Daffner; - Orotrechus pominii Tamanini; $\mathbf{0}$ - Orotrechus biancoi n. sp. 
Cogol dei Siori) gli individui presentano mediamente taglia minore.

Nella popolazione della località tipica (Grotta di Costalta), in tutti gli esemplari maschi dei quali ho potuto esaminare l'edeago, la lamella è sempre troncata inferiormente (Figg. 22, 23), mentre in altre stazioni vi sono individui sia con lamella troncata inferiormente sia con lamella a margine inferiore regolarmente arrotondato (Figg. 26, 27, 34, 35).

Merita maggiore attenzione, invece, il carattere più appariscente tra tutti, il rigonfiamento delle tempie. Troviamo esemplari così conformati, accanto ad altri con capo normale, in varie popolazioni (Figg. 1316) e in modo casuale e sporadico. Nella stazione più occidentale a me nota (Bus del Gobo Onzera) gli individui sono quasi tutti "macrocefali" e rare sono le eccezioni (cfr. Figg. 15 con 13 e 20); probabilmente ciò accade anche al Bus del Bilbom, cavità non lontana e di cui non ho visto reperti.

E' fatto noto, documentato anche in letteratura, che alcune popolazioni, situate ai limiti dell'areale di distribuzione di una specie, presentino caratteri morfologici estremi e una ridotta variabilità che inducono, talvolta, a credere di essere di fronte ad una specie diversa. Sono propenso a considerare in tal senso queste due popolazioni periferiche, nelle quali il carattere autoapomorfo individuale del rigonfiamento delle tempie si estende alla maggioranza degli individui, mentre appare saltuariamente nelle altre stazioni.

Quanto a Orotrechus stephani prenottoi, di cui conosco quattro località e ho esaminato complessiva-

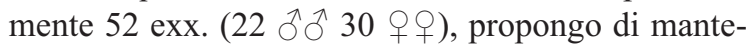
nere il suo status di subspecie valida, sulla base di un accurato studio che ha evidenziato l'assoluta costanza di un importante carattere, la forma della lamella copulatrice dell'edeago, più breve e più larga rispetto alla forma nominale.

Non escludo, tuttavia, l'eventualità che con la scoperta di nuove popolazioni nel Massiccio del Grappa non si debba in futuro rivedere l'assetto tassonomico anche di questa entità.

Di Orotrechus pominii ho esaminato 89 esem-

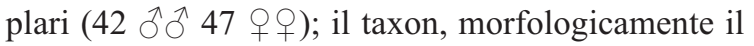
meno specializzato del gruppo, si è rivelato piuttosto variabile, a tutti i livelli, intra- e interpopolazionale, dovuto anche al fatto di occupare un areale molto esteso (i Monti Lessini nel loro insieme) e all'amplissima distribuzione altitudinale (da $330 \mathrm{~m}$ a $1530 \mathrm{~m}$ di quota). Ho potuto osservare, negli esemplari maschi, una tendenza, peraltro non costante (quindi la cito solo come indicativa), a presentare la lamella copulatrice più aperta inferiormente e con l'apofisi basale più allungata nelle popolazioni che man mano si incontrano da est verso ovest.

Considerate le notevoli variazioni proposte ritengo utile riassumerle nella seguente tabella dicotomica:

Superficie dorsale del corpo ricoperta da fitta pubescenza..................... Orotrechus del gruppo stephani

1 Dimensioni mediamente maggiori $(4.03 \div 5.14 \mathrm{~mm}$; media: $4.58 \mathrm{~mm}$ ), il rapporto lunghezza antenne/lunghezza corpo da 0.67 a 0.80 (media: 0.74 ); edeago con armatura del sacco interno a forma di lama appiattita, con parte dorsale ispessita e internamente cava, aperta alla sua base, con margine ventrale debolmente curvato o sub troncato ...............................

1' Dimensioni mediamente minori (3.67 $\div 4.45 \mathrm{~mm}$; media: $3.91 \mathrm{~mm})$, il rapporto lunghezza antenne/lunghezza corpo da 0.61 a 0.70 (media: 0.66 ); edeago con armatura del sacco interno di forma tubolare, bruscamente ripiegato ad angolo quasi retto alla base, che è svasata e munita di un'apofisi ritorta sul lato dorsale

2 Edeago con lamella copulatrice lunga circa quattro volte o più la sua larghezza (cavità veneto-trentine degli Altipiani di Tonezza-Folgaria e Vigolana e dell'Altopiano dei Sette Comuni; un reperto sotto sasso nell'Altopiano dei Sette Comuni) ......

........Orotrechus stephani stephani (Müller, 1913)

2' Edeago con lamella copulatrice lunga circa tre volte o più la sua larghezza (cavità venete del Massiccio del Grappa).....

......Orotrechus stephani prenottoi Daffner, 1986

3 Pronoto lungo circa quanto largo (rapporto lu/la: $0.96 \div 1.04$ ). Apice del lobo mediano dell'edeago ripiegato ventralmente; lamella copulatrice provvista inferiormente di un'espansione obliqua che unisce la base con il suo terzo apicale (cavità vicentine dell'area nota come Bosco del Tretto, pertinente al Gruppo del Carega-Piccole Dolomiti-Pasubio) ....

..Orotrechus biancoi $\mathrm{n} . \mathrm{sp}$.

3' Pronoto trasverso (rapporto lu/la: 0.87 $\div 0.94$ ). Apice del lobo mediano dell'edeago diritto o al più debolmente flesso ventralmente; lamella copulatrice priva di espansione inferiore, ma bruscamente piegata a formare un angolo poco ottuso, quasi retto (cavità veneto-trentine dei Monti Lessini)

Orotrechus pominii Tamanini, 1953 
Orotrechus euganeus perettoi $\mathrm{n}$. ssp.

Diagnosi. Un Orotrechus di dimensioni medio-piccole, ascrivibile, per aspetto, chetotassi pronotale, morfologia dell'edeago e localizzazione geografica, a Orotrechus euganeus Pace, 1974. Distinto dagli altri taxa congeneri berico-lessinei principalmente per alcuni caratteri esterni (Figg. 42-49).

Località tipica. Veneto, Monti Berici. Arcugnano (VI); boschetto a est della Trattoria Moreieta, $335 \mathrm{~m}$ ca.

SERIE TIPICA. Holotypus ${ }^{\lambda}$ : Veneto, Monti Berici. Arcugnano (VI); boschetto a est della Trattoria Moreieta, 335 m ca., 18.VI.1995, E. Piva leg. (CPI). Paratipi, 68 exx. (32 주 36 우우): Veneto, Monti Berici, Arcugnano (VI), boschetto a est della Trattoria Moreieta, $335 \mathrm{~m}$ ca., 4.VI.1995, G. Peretto \& E. Piva leg., 1 ก 3 우 (CPI, MNAV); ibidem, 11.VI.1995, E. Piva leg., 2 우우 (CPI); ibidem, 18.VI.1995, 2 취 dem, 25.VI.1995, E. Piva leg., 1 ภ (CPI); ibidem, 16.VII.1995, E. Piva leg., 1 đ (CPI); ibidem, 26.VIII.1995, D. Mondin \& E. Piva leg., 1 수 2 우 (CPI); ibidem, 3.IX.1995, G. Peretto \& E. Piva leg., 12 ภำ 5 우 (CBI, CPI, MNAV, MUSE); ibidem, 31.III.1996, G. Peretto \& E. Piva leg., 4 त $\widehat{0} 8$ 우 (CBI, CCA, CPI, MNAV); ibidem, 29.VI.1997, E. Piva leg., 1 오 2 우 (CPI); ibidem, 3.V.2009, E. Piva leg., 1 (CPI); ibidem, 8.VI.2012, D. Bianco \& E. Piva leg., 1 \& (CPI); Veneto, Monti Berici, Arcugnano (VI), boschetto a est della Trattoria Moreieta, $335 \div 350$ m ca., 19.IV.1998, E. Piva leg., 4 §ึ $\delta^{\lambda} 4$ 우우 (CGI, CPI); Veneto, Monti Berici, Arcugnano (VI), boschetto a est della Trattoria Moreieta, $350 \mathrm{~m}$, 9.VI.2003, D. Bianco \& N. Righetto leg., 1 q (CBI); Veneto, Monti Berici, Arcugnano (VI), Val Scaranti, scavi lungo il sentiero che percorre la valle, $280 \mathrm{~m}$ ca., 8.III.1997, E. Piva leg., 2 우 (CPI); Veneto, Monti Berici, Arcugnano (VI), Val Scaranti, scavi a quota $120 \mathrm{~m}$, poco sotto la Grotta del Lupo (5524 V/VI), 22.III.2009, E. Piva leg., 1 \& (CPI); Veneto, Monti Berici, Arcugnano (VI), Val Scaranti, scavi a quota 115 $\mathrm{m}$, poco sotto la Grotta del Lupo (5524 V/VI), 5.IV.2009, E. Piva leg., $2 \hat{\jmath}$ (CPI); ibidem, 24.V.2012, E. Piva leg., 2 के Berici, Arcugnano (VI), Grotta Voragine della Moreieta (329 V/VI), 375 m, 18.II.1989, E. Piva leg., 1 † (CPI); ibidem, 16.VI.1989, E. Piva leg., 1 \& (CPI); ibidem, 3.IV.2005, D. Bianco leg., 1 q (CBI); Veneto, Monti Berici, Longare (VI), Voragine Zannini (5952
V/VI), 85 m, 23.II.1997, G. Peretto, E. Piva \& C. Zantedeschi leg., resti di 1 + (CPI).

DeRIVATIO NOMINIS. Dedico la nuova entità al Sig. Giuseppe Peretto (Valdagno, Vicenza), come ringraziamento del piacevole decennio trascorso assieme, indagando innumerevoli cavità del Triveneto e dei Pirenei francesi.

DesCrizione Dell'Holotypus $\widehat{\jmath}$. Corpo robusto (Fig. 42), lucido e glabro, con appendici relativamente brevi, lungo $3.63 \mathrm{~mm}$ (misurato dall'apice delle mandibole all'apice elitrale), di colore testaceo; tegumenti con finissima microscultura a maglie poligonali irregolari.

Capo lungo $0.71 \mathrm{~mm}$ (dal punto più avanzato del margine anteriore del labbro superiore al restringimento collare) e largo $0.61 \mathrm{~mm}$; il rapporto lungh./largh. max. è 1.16. Occhi assenti, tempie poco convesse, glabre, ma con due sole brevi setole in po-

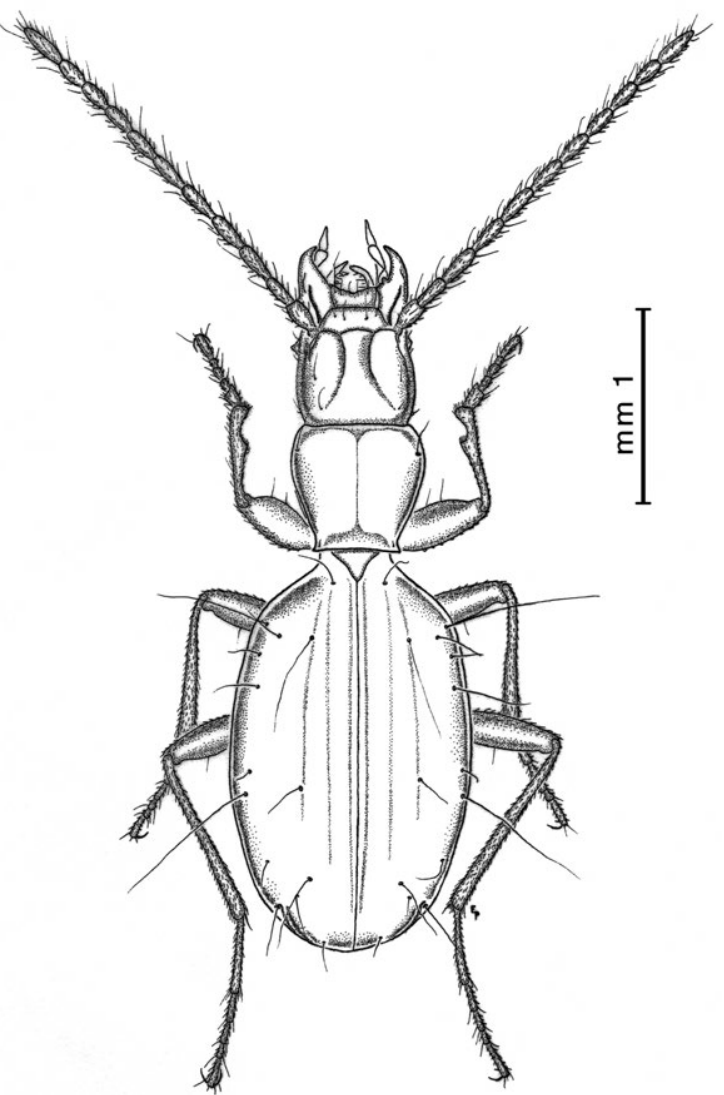

Fig. 42. Orotrechus euganeus perettoi $\mathrm{n}$. ssp., holotypus ${ }^{\lambda}$ : habitus. 
sizione arretrata; solchi frontali completi, bene incisi fino alla seconda setola sopraorbitale, fortemente divergenti in addietro. Presenti due setole sopraorbitali per lato. Antenne lunghe $2.18 \mathrm{~mm}$ (rapporto lungh. antenne/lungh. corpo $=0.60$ ), con articoli corti e spessi. Mandibole brevi e robuste.

Protorace trasverso, lungo $0.64 \mathrm{~mm}$ e largo $0.70 \mathrm{~mm}$ (rapporto lu/la: 0.91), a lati regolarmente arrotondati nel terzo anteriore, poi gradatamente ristretti in linea lievemente ricurva fin quasi alla base, dove sono brevemente ma nettamente sinuati; angoli posteriori acuti e salienti all'esterno. Doccia marginale stretta e regolare. Setole pronotali presenti: le anteriori, di normale sviluppo, sono inserite nel primo quarto anteriore del protorace; le basali, situate agli angoli posteriori, sono molto brevi ed esili.

Elitre ovali, lunghe poco più di una volta e mezza la loro larghezza massima (rapporto lu/la, considerate insieme: 1.60 ), molto convesse, soprattutto anteriormente, con omeri arrotondati; doccia laterale di modesto sviluppo, carena apicale breve ma evidente. Stria suturale profondamente impressa per quasi tutta la sua lunghezza, le strie seconda e terza poco incise, ma parzialmente distinguibili sul disco elitrale; le altre svanite. Chetotassi: setola scutellare bene sviluppata; serie discale di due setole, inserite sulla terza stria: l'anteriore appena dopo il livello della prima della serie ombelicata, la posteriore tra i livelli delle setole quinta e sesta della serie ombelicata. Gruppo omerale della serie ombelicata con la prima setola più vicina alla seconda che non alla terza, quest'ultima equidistante dalla seconda e dalla quarta. Gruppi mediano e apicale senza caratteristiche particolari. Triangolo apicale completo, con la setola esterna piuttosto ravvicinata a quella anteriore.

Zampe robuste, con tibie e tarsi pubescenti; primo protarsomero dilatato e dentato internamente.

Apparato copulatore: edeago lungo $0.53 \mathrm{~mm}$, misurato dalla base all'apice. In visione laterale (Fig. 43) il lobo mediano risulta poco incurvato, affusolato, con bulbo basale poco evidente; il margine ventrale è debolmente curvato e l'apice è diritto, smussato all'estremità. In visione dorsale (Fig. 44) presenta l'apice largo e arrotondato. Parameri ampi, piegati verso la metà a formare una costola convessa, a sua volta seguita da un lobo incavato a cucchiaio, muniti di numerose setole: quattordici (il sinistro) e undici (il destro), disposte in numero di quattro lungo la costola, le restanti distribuite in modo irregolare sulla superfi- cie del lobo concavo. Endofallo con lamella copulatrice stretta e allungata, appena ritorta ventralmente all'apice, debolmente rialzata alla base (Figg. 45 e 46).

DESCRIZIONE DEI PARATIPI E VARIABILITÀ. Lunghezza del corpo (dall'apice delle mandibole all'apice elitrale): $3.56-3.99 \mathrm{~mm}$ nei ${ }^{\circ}$ oै (media: $3.77 \mathrm{~mm}$ ), $3.50-4.02 \mathrm{~mm}$ nelle 우 (media: $3.74 \mathrm{~mm}$ ). Il rapporto lunghezza an-

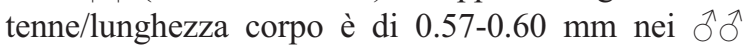
(media: 0.58), di 0.55-0.61 nelle 우 (media: 0.58).

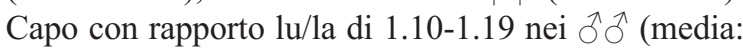
1.15), di 1.09-1.19 nelle 우우 (media: 1.14) (misurando la lunghezza del capo dal punto più avanzato del mar-
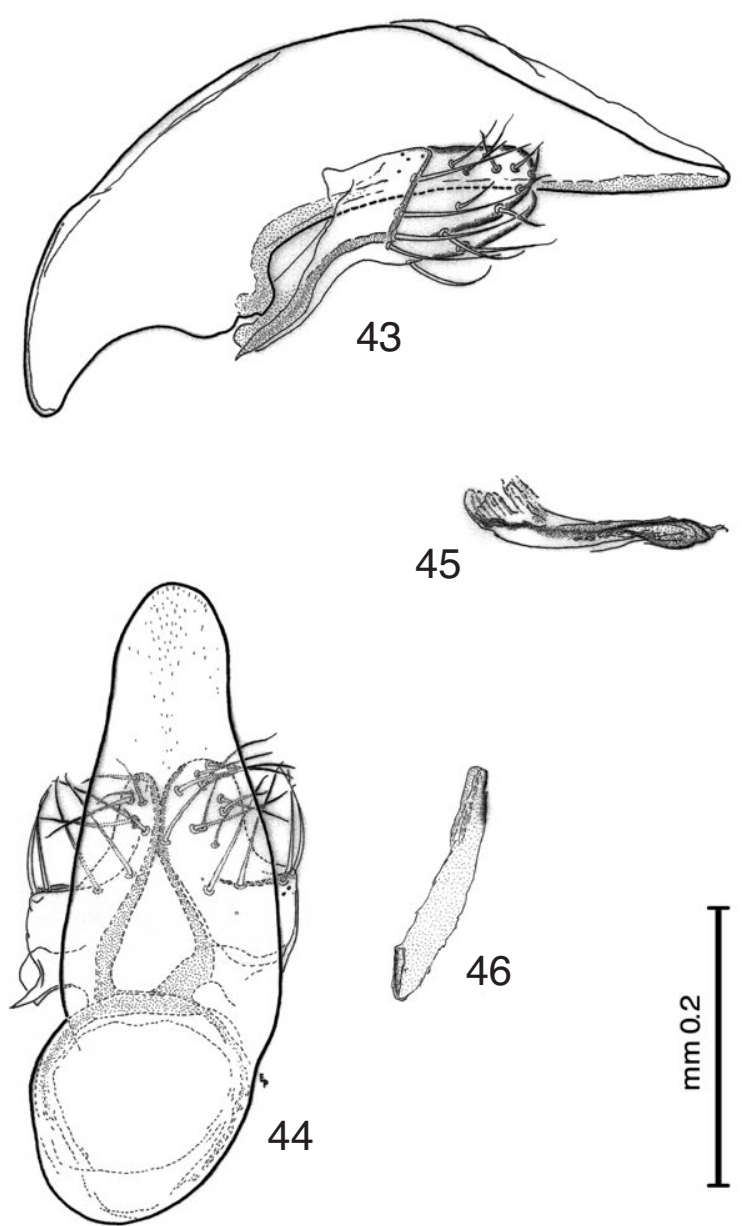

Figg. 43-46. Orotrechus euganeus perettoi n. ssp., holotypus $\delta: 43$. edeago, in visione laterale; 44 . idem, in visione dorsale; 45. lamella copulatrice, in visione laterale; 46. idem, in visione dorsale. 
gine anteriore del labbro superiore al restringimento collare). Il protorace, trasverso, ha un rapporto lu/la di 0.880.96 in ambo i sessi (medie: 0.91 nei $\widehat{\partial} \hat{o}, 0.90$ nelle 우); lati sinuati prima degli angoli posteriori, questi acuti e sporgenti all'infuori. Setole basali generalmente come nell'olotipo, ma talvolta più lunghe e bene evidenti; solo in due casi esse non sono visibili (probabilmente per caduta e non per atrofia). Elitre con rapporto lunghezza/larghezza massima (considerate insieme) di 1.51-1.68. Chetotassi: primo poro della serie discale oltre il livello del secondo della serie ombelicata, tranne in un caso in cui è posizionato appena prima. Un'unica eccezione anche per il primo poro della serie ombelicata posizionato prima del secondo, mentre è inserito oltre in tutti gli altri paratipi. Il secondo poro discale, contrariamente all'olotipo, si trova quasi sempre prima del quinto della serie omerale e raramente allo stesso livello o dopo di questo. Infine, la distanza tra i pori quinto e sesto della serie ombelicata è inferiore a quella tra i pori secondo e terzo, tranne in un caso in cui tale distanza è maggiore $\mathrm{e}$ in un altro caso, dove invece è pressoché uguale. Nei maschi l'apparato copulatore presenta il lobo mediano conforme a quello dell'olotipo; poco significativi risultano il maggiore o minore numero di setole dei parameri e le lievi differenze nelle dimensioni e nella forma della lamella copulatrice. Armatura genitale femminile come da Fig. 47: gonostili (Figg. 48 e 49) arrotondati all'apice, provvisti di due setole tergali, di cui una sempre molto robusta; fossetta preapicale con due setole e due bastoncelli sensoriali. In un paratipo femmina uno dei due gonostili presenta una terza setola tergale. Parti laterali degli emisterniti munite di numerose setole premarginali e marginali.

DistribuZione, ECOLOGiA. Evidentemente legato all'ambiente sotterraneo superficiale (MSS), Orotrechus euganeus perettoi $\mathrm{n}$. ssp. è stato raccolto casualmente e raramente anche in grotta (Grotta Voragine della Moreieta e Voragine Zannini). La prima delle due cavità è situata alla sommità di un colle che verso est supporta la nota Trattoria omonima, per poi insistere su un versante di matrice calcarea fino a raggiungere una vallecola, al di là della quale si estende una zona a suolo di origine vulcanica, località tipica del nuovo taxon (il boschetto a est della Trattoria Moreieta; Fig. 50).

Da quanto osservato durante le ricerche, è apparsa subito evidente una maggiore presenza di individui nella zona non calcarea, dove peraltro difettavano predatori tipici del comprensorio berico, quali il Cara- bide Pterostichino Speluncarius bericus Monguzzi, 1982 (nessun reperto in questo substrato) e lo Stafilinide Lathrobium (Glyptomerus) alzonai Capra \& Binaghi, 1938 (complessivamente solo un adulto e tre larve in tale suolo), mentre nella zona calcarea sono stati raccolti solo due esemplari di Orotrechus euganeus perettoi $\mathrm{n}$. ssp., a fronte di numerosi (frequenti, ma non comuni) reperti degli altri due predatori.

Molto probabilmente la composizione litologica dei due ambienti ha influito sulla presenza/assenza di una specie rispetto all'altra, ma sembra altrettanto plausibile una correlazione, di tipo competitivo e di reciproca esclusione, fra le diverse entità che vivono in questi habitat.

Da ricordare, infine, che nelle cavità dei Monti
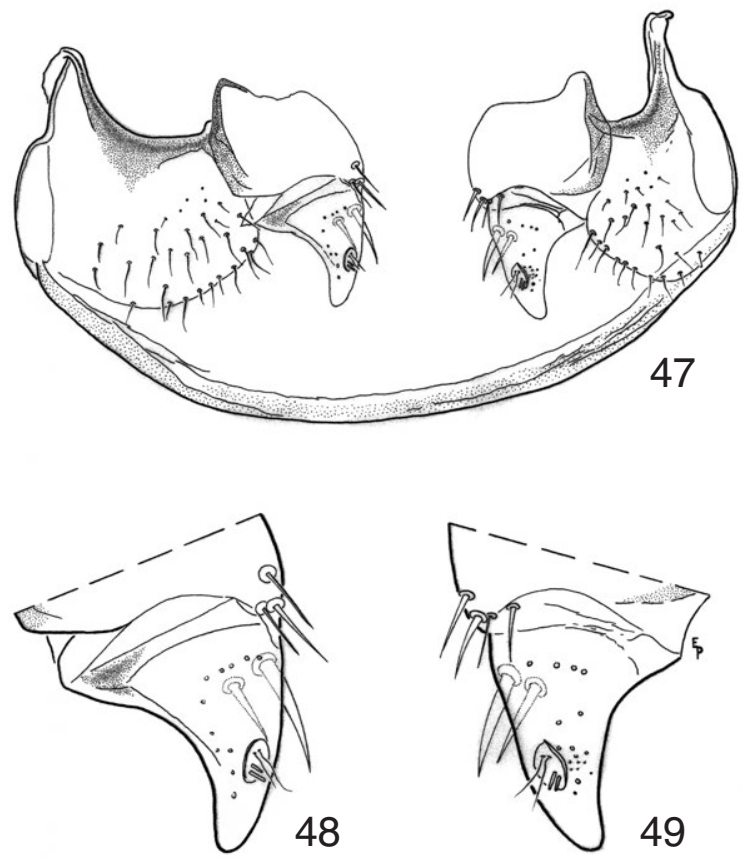

$0.2 \mathrm{~m} \mathrm{~m}$ : fig. 47

$0.2 \mathrm{~m} \mathrm{~m}$ :figg. 48,49

Figg. 47-49. Orotrechus euganeus perettoi n. ssp., paratypus † (boschetto a est della Trattoria Moreieta, località tipica): 47. armatura genitale femminile in visione ventrale; 48-49. gonostili a maggiore ingrandimento. 
Berici (quindi anche nella Grotta Voragine della Moreieta) vive un altro elemento endemico, molto specializzato: si tratta di Orotrechus fabianii (Gestro, 1900). Segnalo, per questa specie, un dato inedito: il rinvenimento, in ambiente sotterraneo superficiale (MSS), di un esemplare in una trappola a caduta posi- zionata in fondo alla valletta che nel boschetto a est della Trattoria Moreieta divide le due zone a diversa litologia. La citazione del ritrovamento di questa entità al di fuori dell'ambiente grotta, apparsa per la prima volta in Tamanini (1.c., 1953b, pag.60: testualmente “...In diverse grotte ed anche all'esterno sotto le pietre

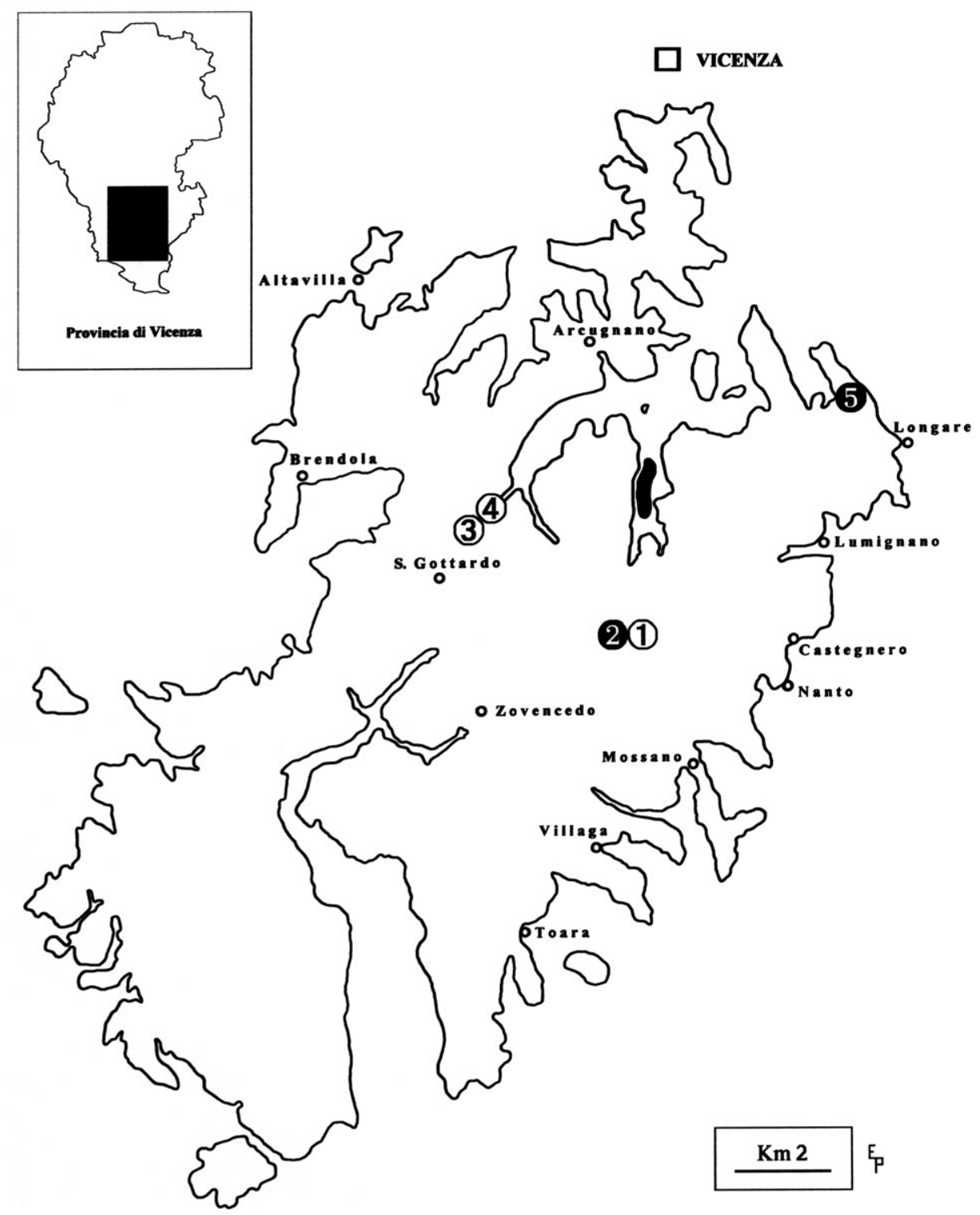

Fig. 50. Cartina di distribuzione di Orotrechus euganeus perettoi n. ssp. Stazioni endogee: (1) boschetto a est della Trattoria Moreieta, località classica; 2 Grotta Voragine della Moreieta; (3) Val Scaranti, scavi lungo il sentiero che percorre la valle; (4) Val Scaranti, poco sotto la Grotta del Lupo. Stazioni di ambiente grotta; 5 Voragine Zannini. 
molto infossate nei Monti Berici...") e poi ripresa da altri autori (Porta, 1959; Vigna Taglianti, 1982) non trova alcun riscontro nella letteratura precedente, nonostante la personale accurata ricerca bibliografica, condotta partendo dalla descrizione del taxon.

Le altre due stazioni di raccolta della nuova entità sono la Val Scaranti (lungo il sentiero che la percorre nel versante sud e nell'incisione valliva, a poca distanza dalla Grotta del Lupo) e la Voragine Zannini (dove sono stati raccolti i resti di un esemplare).

\section{OSSERVAZIONI}

Il genere Orotrechus Müller, in base a recenti studi sulla filogenesi molecolare dei Trechini (Faille et al., 2013), oltre che essere confermato come monofiletico, risulta di estrema complessità, sia morfologicamente, presentando la più vasta gamma di modificazioni in relazione all'ambiente sotterraneo (da specie endogee di piccole dimensioni a facies "anoftalma", a specie più grandi, eucavernicole, a facies "afenopsiana"), sia dal punto di vista corologico, con innumerevoli casi di simpatria e/o sintopia di elementi attribuibili a differenti gruppi di specie e insediatisi nei vari siti in tempi diversi; inoltre, è da evidenziare l'aspetto ecologico, poiché, in pratica, tutti i microambienti sotterranei, siano essi naturali o artificiali, prossimi alla superficie o ipogei profondi, a bassa o ad alta quota, sono colonizzati da una o più specie del genere.

Ciò detto, concordo con Faille et al. (2013) nell'avvertire l'esigenza di una revisione tassonomica del genere Orotrechus, definendo quali entità attribuire all'uno o all'altro gruppo di specie.

\section{RINGRAZIAMENTI}

Ringrazio i miei collaboratori, che a diverso titolo hanno contribuito a radunare importante materiale: Giuseppe Peretto, per anni compagno di ricerche sul campo; Dante Bianco, attivissimo ricercatore di Artropodi ipogei; i soci del Gruppo Grotte CAI di Valdagno Francesca Casa, Moreno Cocco, Luca Dalle Tezze, Alessandro De Munari, Nicola Righetto, Daniela Sbicego, Michele Tomasi, Paolo Urbani e Giuseppe Zarantonello, per l'attiva collaborazione alle ricerche. Sono grato, inoltre, a Massimo Eder e ad Alessandra Franceschini, del Museo delle Scienze (MUSE) di Trento, per il prestito di materiale tipico, ad Achille Casale e Pier Mauro Giachino, per i consigli avuti.

\section{BIBLIOGRAFIA}

AgAzZI G., 1959 - Ricerche biospeleologiche nei Lessini sud-orientali. Il maschio dell'Orotrechus vicentinus Gestro (Coleoptera, Trechidae). Bollettino della Società entomologica italiana, 89(1-2): 24-28.

Bucciarelli I., 1957 - Un interessante reperto faunistico sui Colli Berici. La $q$ del Lathrobium (Glyptomerus) alzonai Capra e Binaghi (Col. Staphylinidae). Bollettino della Società entomologica italiana, 87(5-6): 81-83.

Bucciarelli I., 1958 - La larva del Lathrobium (Glyptomerus) alzonai Capr. Bin. (Col. Staphylinidae). Bollettino del Museo Civico di Venezia, 11: 29-34.

CAPra F., Binaghi G., 1938 - Un nuovo Glyptomerus dei Monti Berici. Appunti sulla morfologia degli uriti $8^{\circ}$ e $9^{\circ}$ e sulla meccanica dell'estroflessione dell'edeago nei Lathrobium (Col. Staphylinidae). Bollettino della Società entomologica italiana, 70: 130-135.

DAfFner H., 1986 - Orotrechus prenottoi sp. n. von den Prealpi Venete-Norditalien (Coleoptera, Carabidae, Trechinae). Acta Coleopterologica, 1(2): 29-36.

DAFFner H., 1987 - Orotrechus martinellii spec. nov. vom Monte Baldo-Norditalien (Coleoptera, Carabidae, Trechinae). Nachrichtenblatt der Bayerischen Entomologen, 36(2): 39-43.

Faille A., Casale A., Balke M., Ribera I., 2013 - A molecular phylogeny of Alpine subterranean Trechini (Coleoptera: Carabidae). BMC Evolutionary Biology, 13: 248-263.

Gestro R., 1900 - Gli anoftalmi trovati finora nel Veneto. Annali del Museo Civico di Storia Naturale di Genova, 40: $567-572$. Gestro R., 1907 - Una gita in Garfagnana. Annali del Museo Civico di Storia Naturale di Genova, serie 3a, Vol. 3(43): 168-177.

JEANNEL R., 1928 - Monographie des Trechinae (Troisième livraison). Les Trechini cavernicoles. L'Abeille, 35: 1-808.

Monguzzi R., 1982 - Speluncarius bericus, nuova specie cavernicola dei Colli Berici (Vicenza) ed osservazioni su S. stefani (Jur.) (Coleoptera Carabidae Pterostichinae). Giornale italiano di Entomologia, 1: 49-57.

MÜLlER J., 1913 - Drei neue blinde Trechen aus Oesterreich. Entomologische Blätter, IX: 299-303.

Müller G., 1928 - Il genere Orotrechus Müll., Jeannel. Bollettino della Società entomologica italiana, Genova, 60: 92-98. 
MÜLLER G., 1930 - I coleotteri cavernicoli italiani. Le Grotte d'Italia, 4(2): 65-85.

Müller G., 1933 - Un nuovo Orotrechus dei dintorni di Rovereto. Studi Trentini di Scienze Naturali, 14: 114-117.

PACE R., 1974 - Descrizione di un nuovo Orotrechus G. Müller dei Colli Euganei (Coleoptera Trechidae). Memorie del Museo Civico di Storia Naturale di Verona, 20(1972): 495-501.

PIVA E., 1983 - Descrizione del maschio di Orotrechus pominii Tamanini, 1953 (Coleoptera Carabidae Trechinae). Giornale italiano di Entomologia, 1: 289-294.

Pomini F.P., 1940 - Una nuova specie di Orotrechus (O. Juccii) delle Prealpi Veronesi (Coleopt. Carabidae). Bollettino della Società entomologica italiana, 72 (5-6): 81-86.

PoRTA A., 1923 - Fauna Coleopterorum Italica. I. Piacenza, pp. 1-285.

Porta A., 1934 - Fauna Coleopterorum Italica. Supplementum. Piacenza, pp. 1-208.

Porta A., 1949 - Fauna Coleopterorum Italica. Supplementum II. Sanremo, pp. 1-386.

PorTA A., 1959 - Fauna Coleopterorum Italica. Supplementum III. Sanremo, pp. 1-344.

TAMAnini L., 1937 - Nota sull'Orotrechus Stephani Müll. sbsp. roboretanus Müll. e descrizione del maschio. Studi Trentini di Scienze Naturali, 18: 79-84.

TAMAnINI L., 1953a - Gli Orotrechus dei Lessini e descrizione di due nuove forme (Coleoptera, Trechidae). Memorie del Museo Civico di Storia Naturale di Verona, 4: 13-24.

TAMAnini L., 1953b - Gli “Orotrechus” delle Prealpi Veneto-Trentine (Coleoptera, Trechidae). Studi Trentini di Scienze Naturali, 30: 34-64.

Vigna Taglianti A., 1981 - Un nuovo Orotrechus delle Prealpi Venete (Coleoptera, Carabidae). Bollettino del Museo Civico di Storia Naturale di Verona, 7(1980): 69-84.

Vigna TAglianti A., 1982 - Le attuali conoscenze sui Coleotteri Carabidi cavernicoli italiani. Lavori della Società Italiana di Biogeografia, nuova serie, Vol. VII (1978): 339-430.

Vigna Taglianti A., 1993 - Coleoptera Archostemata, Adephaga 1 (Carabidae). In: Minelli A., Ruffo S., La Posta S. (eds.) Checklist delle specie della fauna italiana, 44. Calderini, Bologna. 